\title{
Reconstruction of air-shower measurements with AERA in the presence of pulsed radio-frequency interference
}

\author{
Tim Huege $\mathrm{e}^{1,4, \star}$, Christoph B. Welling ${ }^{2}$ for the Pierre Auger Collaboration ${ }^{3, \star \star}$ \\ ${ }^{1}$ Karlsruhe Institute of Technology, Institute for Nuclear Physics, Karlsruhe, Germany \\ ${ }^{2}$ RWTH Aachen University, Aachen, Germany \\ ${ }^{3}$ Observatorio Pierre Auger, Av. San Martín Norte 304, 5613 Malargüe, Argentina \\ ${ }^{4}$ also at: Vrije Universiteit Brussel, Brussels, Belgium
}

\begin{abstract}
The Auger Engineering Radio Array (AERA) is situated in the Argentinian Pampa Amarilla, a location far away from large human settlements. Nevertheless, a strong background of pulsed radio-frequency interference (RFI) exists on site, which not only makes radio self-triggering challenging but also poses a problem for an efficient and pure reconstruction of air-shower measurements. We present how our standard event reconstruction exploits several strategies to identify and suppress pulsed noise, and quantify the efficiency and purity of our algorithms. These strategies can be employed by any experiment taking radio data in the presence of pulsed RFI.
\end{abstract}

\section{Introduction}

AERA consists of 153 radio-detector stations covering a total area of $17 \mathrm{~km}^{2}$ in the Pampa Amarilla near Malargüe, Argentina. Although situated in a rural area, a strong background of pulsed RFI is present at the site. Investigations of its origin were not entirely conclusive, but it is clear that the pulses arise from multiple sources: power lines, faulty transformers in a nearby village, communication towers, oil rigs, and others. The average rate of background pulses above a signal-to-noise threshold of 10 amounts to $15 \mathrm{kHz}$, and the pulse characteristics are generally similar to signals emitted by extensive air showers, making, in particular, the self-triggering on radio signals very challenging.

While the exploitation of external triggering from other detector components of the Pierre Auger Observatory provides an efficient and pure trigger, the pulsed RFI also poses a challenge for successful event reconstruction. In the following, we first describe how we exploit the hybrid nature of our measurements in the event reconstruction to mitigate the adverse effects of pulsed RFI. Afterwards, we describe our strategies to identify and reject pulsed RFI disturbing the measurements in individual AERA antenna stations, which improves both the efficiency and purity of our reconstruction.

\section{Hybrid reconstruction strategy}

The Pierre Auger Observatory is a hybrid detector measuring individual air showers with complementary detection techniques [1]. The AERA events triggered by the Auger surface detector (SD) also

${ }^{\star}$ e-mail: tim.huege@kit.edu

${ }^{\star \star}$ Full author list at http://www.auger.org/archive/authors_2018_06.html 


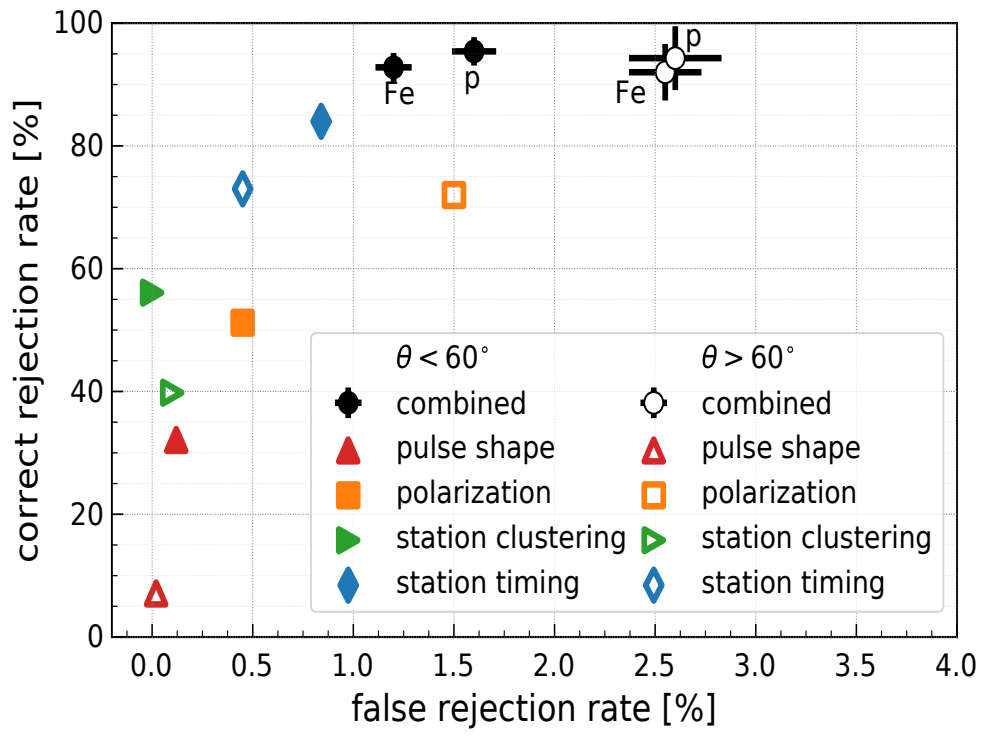

Figure 1. Performance of the individual rejection strategies for air showers with zenith angles below (filled symbols) and above $60^{\circ}$ (open symbols). The black points illustrate the performance of all strategies combined in the same reconstruction.

have an associated SD measurement. Reconstruction of the SD data provides the event geometry, i.e., the orientation and impact point of the air-shower axis. Using this information early on in the radio reconstruction, we calculate for each AERA antenna station the expected time of arrival of the radio pulse. Uncertainties in the SD-reconstructed event geometry are propagated to set the width of the "signal-search window", which typically amounts to $~ 1000 \mathrm{~ns}$.

This strategy is vastly superior to searching for radio pulses in the whole recorded radio trace of length $10 \mu \mathrm{s}$, which for $15 \mathrm{kHz}$ of RFI pulses has a probability of $15 \%$ to harbor an RFI pulse in each individual antenna station. As typically more than 100 antenna stations are read out on an SD trigger, this on average would yield $\sim 15$ stations with RFI pulses, while cosmic-ray pulses are typically only present in 3-5 AERA stations [2].

\section{Station-level suppression of RFI pulses}

With a signal search window of $\sim 1000 \mathrm{~ns}$, the chance probability of picking up an RFI pulse shrinks to $\sim 1.5 \%$. For more than 100 stations read out, typically one or two RFI pulses remain in the data used for reconstruction. These pulses deteriorate the reconstruction efficiency and purity. We have thus developed several strategies to identify and suppress these RFI pulses.

The strategies have been evaluated by performing a standard event reconstruction on CoREAS simulations [3] which have undergone a complete detector simulation [4] and have been added to measured noise. An RFI pulse correctly identified as RFI is denoted a "correct rejection" in the following. Correspondingly, a "false rejection" characterizes a cosmic-ray pulse identified as RFI. For each strategy discussed in the following, we evaluate the fraction of correct versus false rejections individually before combining all algorithms and evaluating the overall performance.

Pulse shape: Radio pulses from air showers measured in the 30 to $80 \mathrm{MHz}$ band are typically bandwidth-limited with a characteristic width and shape, oscillating with a frequency of $55 \mathrm{MHz}$. We 
define two thresholds: a fraction $a$ of the peak amplitude in the signal-search window, and a multiple $b$ of the trace RMS in a noise window before the arrival of the pulse. The algorithm counts the upward crossings $N$ of these two thresholds in a given time window $\Delta t$ centered on the pulse. Pulses with more crossings than a threshold $N_{\max }$ are too long and thus rejected as RFI. After optimization of the parameters $a, b, \Delta t$ and $N_{\max }$, the performance is shown in the red triangles in Fig. (1).

Signal polarization: Radio pulses from air showers have specific polarization characteristics arising from the superposition of geomagnetic and charge-excess radiation (see, e.g., [5]). Using the event geometry provided by the SD reconstruction (including its uncertainties), the actual pulse polarization at each antenna station is compared with the expected polarization. If the deviation is larger than a defined threshold, the pulse is considered RFI and is rejected. The performance is demonstrated by the yellow squares in Fig. (1).

Station clustering: The radio-emission footprint covers a contiguous area. Consequently, isolated antenna stations with a detected pulse have likely measured RFI and are rejected from the reconstruction. The performance of this algorithm is illustrated by the green triangles in Fig. (1).

Station timing: The arrival times of radio pulses from an air shower form a wavefront. We perform a bottom-up reconstruction which first fits the radio-pulse arrival times of the three antenna stations nearest the SD core with a plane wave and then subsequently adds antenna stations further out to the fit. If adding a particular antenna station decreases the $\chi^{2}$ probability of the fit below $5 \%$, that station is rejected - its pulse arrival time does not match that of the other pulses. The performance is illustrated by the blue diamonds in Fig. (1).

Overall performance: We now combine all of these strategies in the reconstruction and apply them to a data set of simulations with both proton and iron primaries. The overall performance is shown in the black points in Fig. (1). Approximately $90 \%$ of the RFI pulses are rejected while only $\sim 1.5 \%$ of the radio pulses from vertical and $\sim 2.5 \%$ of the radio pulses from inclined air showers are rejected. There are only small differences in the performance for proton- and iron-induced air showers, i.e., the RFI-rejection algorithms do not introduce a significant selection bias.

Validation with measured data: We have validated the performance of the combination of these algorithms also with measured data. For each measured air shower, we determine the probability for successful reconstruction using the SD-reconstructed geometry and energy as input for an analytical model [6]. We find that for events for which no successful detection is expected, the fraction of reconstructed versus triggered events drops by a factor of $\sim 6$ when applying the RFI rejection. This illustrates that in particular the purity of our reconstruction is significantly increased.

\section{Conclusions}

We have presented how we achieve efficient and pure reconstruction of radio data taken in the presence of strong pulsed RFI by exploiting hybrid measurements. The strategies employed here can also be of use to other radio-detection projects measuring in noisy environments.

\section{References}

[1] A. Aab et al. (Pierre Auger Collaboration), Nucl. Instrum. Meth. A 798, 172 (2015)

[2] A. Aab et al. (Pierre Auger Collaboration), Phys. Rev. D 93, 122005 (2016)

[3] T. Huege, M. Ludwig, C.W. James, AIP Conf. Proc. pp. 128-132 (2013)

[4] P. Abreu et al. (Pierre Auger Collaboration), Nucl. Instr. Meth. A 635, 92 (2011)

[5] T. Huege, Physics Reports 620, 1 (2016)

[6] C. Glaser, S. de Jong, M. Erdmann, J.R. Hörandel, Astropart. Phys., arXiv : 1806.03620 